\title{
Influência do uso do solo na ocorrência e diversidade de FMAs em Latossolo no Sul do Brasil
}

\section{The influence of soil use on the occurrence and diversity of AMFs in a oxisol from Southern Brazil}

\author{
Rodrigo Ferreira da Silva ${ }^{1 *}$; Rudinei De $\mathrm{Marco}^{2}$; Gilvan Moisés Bertollo ; \\ Márcia Matsouka ${ }^{1}$; Diego Ricardo Menegol $^{4}$
}

\section{Resumo}

O uso do solo e fatores edáficos podem alterar a ocorrência e distribuição de fungos micorrízicos arbusculares. O objetivo do trabalho foi determinar a influência de diferentes usos do solo na comunidade de fungos micorrízicos arbusculares (FMAs). As áreas analisadas foram de floresta nativa, área de pousio, pastagem intensiva, pastagem extensiva, plantio direto, plantio convencional, pomar de laranja, videira e Eucalyptus dunnii. A partir da coleta de amostras de solo estabeleceu-se um cultivo armadilha com Brachiaria brizantha para a recuperação de espécies de FMAs, conduzida em câmara BOD em delineamento inteiramente casualizado, com quatro repetições. Após três meses do cultivo armadilha, os esporos foram extraídos pelo método de peneiramento úmido e centrifugação em sacarose e identificados por meio de suas características morfológicas. Foram determinadas a abundância de esporos por espécie e por área avaliada, índice de riqueza de Margalef, equitabilidade de Pielou, dominância de Simpson e diversidade de Shannon. Os resultados revelaram a ocorrência de Glomus mosseae, Glomus aggregatum, Glomus clarum, Acaulospora P., e Scutellospora reticulata. O gênero Glomus foi o mais abundante nas áreas analisadas. A abundância de esporos de FMAs é maior no plantio direto. $\mathrm{O}$ pH do solo afeta a ocorrência de espécies de FMAs. A floresta nativa apresentou a menor dominância e maior diversidade de espécies de FMAs, quando comparada com as áreas submetidas a diferente uso do solo.

Palavras-chave: Eucalipto, floresta nativa, plantio direto, pomar de laranja, videira

\begin{abstract}
The soil use and edaphic factors can to change arbuscular mycorrhizal fungi occurrence and distribution. The objective of this study was to determine the influence of different soil uses in the arbuscular mycorrhizal fungi (AMF) community. The areas of native Forest, fallow area, intensive grassland, extensive grazing, no-tillage, conventional tillage, orange orchard, vines and Eucalyptus dunnii were analyzed. From de collection of soil samples was established trap cultivation with Brachiaria brizantha for recovering AMF species that was conducted in camera BOD in casualized lineation, with four
\end{abstract}

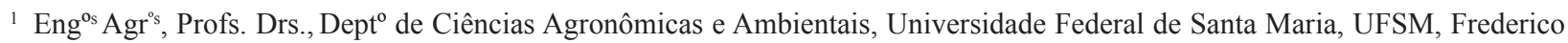
Westphalen, RS.E-mail: rofesil@bol.com.br; marciamatsuoka@yahoo.com.br

2 Eng $^{\circ}$ Florestal, Discente de Mestrado do Programa de Pós-Graduação em Agronomia: Agricultura e Ambiente, PPGAAA, UFSM, Frederico Westphalen, RS. Bolsista CAPES. E-mail: rudineidemarco@hotmail.com

${ }^{3}$ Eng $^{\circ}$ Agr $^{\circ}$, Discente de Mestrado do Programa de Pós-Graduação em Agronomia: Agricultura e Ambiente, PPGAAA, UFSM, Frederico Westphalen, RS. Bolsista CAPES. E-mail: gilvanbertollo@yahoo.com.br

${ }^{4} \mathrm{Eng}^{\mathrm{o}} \mathrm{Agr}^{\mathrm{o}}$, M.e em Agronomia, Agricultura e Ambiente, Laboratorista do Dept ${ }^{\mathrm{o}}$ de Ciências Agronômicas e Ambientais, UFSM, Frederico Westphalen, RS. E-mail: menegoldr@gmail.com

* Autor para correspondência 
repetitions. After three months of trap cultivation, spores were extracted by the method of wet sieving and centrifugation on sucrose and identified by their morphological characteristics. We determined the abundance of spores by species and evaluated area, Margalef richness index, Pielou equitability, Simpson dominance and Shannon diversity. The results revealed the occurrence of Glomus mosseae, Glomus aggregatum, Glomus clarum, Acaulospora sp. and Scutellospora reticulata. The genus Glomus was the most abundant in the areas analyzed. The abundance of spores of AMF is greatest in no-tillage. The soil $\mathrm{pH}$ affects the occurrence of AMF species. The native forest showed lower dominance and diversity of AMF species than areas subjected to different soil use.

Key words: Eucalyptus, native forest, no-tillage, orange orchard, vines

\section{Introdução}

Os benefícios dos microrganismos para o ambiente edáfico e para as plantas possibilitam a sustentabilidade de agroecossistemas, seja pelos ciclos biogeoquímicos, pela formação do solo ou aumento da absorção de nutrientes (MENDES; REIS JUNIOR, 2010). Em meio aos sistemas biológicos inclui-se a simbiose entre fungos micorrízicos e as raízes das plantas (SCHIAVO; MARTINS, 2002). Nesta simbiose, o fungo por meio de suas hifas promove aumento da superfície específica de absorção da raiz no solo (BALOTA; MACHINESKI; STENZ, 2009). Os fungos micorrízicos arbusculares (FMAs) constituem um importante grupo de microrganismos do solo e da rizosfera, tornando-se de grande interesse ecológico e agrícola (ARAÚJO, 2008). Os principais benefícios da associação micorrízica nas espécies vegetais ocorrem pelo aumento da capacidade de absorção e utilização de nutrientes pelas hifas, especialmente os de baixa mobilidade (RAMOS et al., 2012), maior tolerância das plantas micorrizadas a metais pesados presentes no solo, estresse hídrico e a microrganismos patógenos de plantas (GUO et al., 2013; SMITH; READ, 2008).

Conforme Fernandes (2009) a população de FMAs está relacionada a fatores edáficos, climáticos e de plantas por promover influência em sua distribuição e ocorrência. Fernandes (2009) comenta ainda, que as práticas de manejo e uso do solo também podem alterar a composição quantitativa e qualitativa desses fungos no solo. Gomide (2013) relatou que os aspectos ecológicos de vegetação podem explicar melhor a diversidade de FMAs do que os atributos químicos do solo, pois é uma tentativa de demonstrar os efeitos de processos locais atuando sobre a diversidade destes fungos no solo. Nesse sentido, a rotação de culturas e a maior diversidade de espécies de plantas em um ecossistema poderá promover aumento na população dos FMAs nativos do solo, beneficiando as plantas de interesse agrícola e florestal e os cultivos subsequentes.

O conhecimento da diversidade das populações de FMAs, seu papel e interações com o meio são considerados requisitos para o estabelecimento de manejo que permita o aumento no crescimento da planta (ANTONIOLLI, 1999). Nesse sentido, Ramos et al. (2012) relataram que o estudo da diversidade de FMAs, em condições de campo, pode ser realizado por meio de métodos qualitativos e quantitativos de esporos e da associação micorrízica. O objetivo do trabalho foi determinar a Influência do uso do solo na ocorrência e diversidade de FMAs em Latossolo no Sul do Brasil.

\section{Material e Métodos}

As amostras de solo utilizadas para a identificação e contagem de esporos dos FMAs foram coletadas em maio de 2013 nos municípios de Taquaruçu do Sul e Frederico Westphalen, na região Norte do Estado do Rio Grande do Sul, em Latossolo Vermelho aluminoférrico, de textura argilosa (EMBRAPA, 2006). De acordo com a classificação de Köppen, o clima da região é do tipo Cfa caracterizado com precipitação anual variando de 1900 a 2200 mm (ALVARES et al., 2013). 
Foram coletadas amostras de solo formadas por dez subamostras, na profundidade de 0 a 20 cm com auxílio de um trado holandês, com quatro repetições, sendo cada repetição considerada uma unidade amostral. O delineamento experimental foi o inteiramente casualizado com nove tratamentos, correspondendo a diferentes usos do solo: Floresta nativa $(\mathrm{FN})$ - representado por um fragmento de Floresta Estacional Decidual com estágio sucessional avançado; Pousio (P) - área com estágio sucessional inicial da vegetação nativa; Pastagem Intensiva (PI) - área destinada à pastagem rotacionada com Tifton 85 (Cynodon sp.); Pastagem extensiva (PE) - área sem manejo utilizada para o pastoreio de bovinos de corte, com gramínea (Axonopus sp.); Plantio direto (PD) - área há cinco anos em sistema de plantio direto de soja e trigo; Plantio convencional (PC) - cultivo de milho e fumo há cinco anos sob preparo convencional (com revolvimento total do solo); Pomar de Laranja (PL) - cultivada há doze anos com laranja valência (Citrus sinensis); Videira (V) - área há cinco anos com videira (Vitis sp.); e Eucalyptus dunnii (Ed) - área há oito anos com Eucalyptus dunnii, no espaçamento $2 \times 2 \mathrm{~m}$.

Para a recuperação de espécies de FMAs, que não estavam esporulando no momento da coleta, encontrando-se em estágio de hifa ou outro propágulo, estabeleceu-se um cultivo armadilha com Brachiaria (Brachiaria brizantha). O cultivo foi instalado em câmara BOD com fotoperíodo (16 horas luz e 8 horas escuro), em vasos de $500 \mathrm{~cm}^{3}$, contendo solo das quatro repetições obtidas a campo. Aos 30, 45 e 60 dias de cultivo da Brachiaria, cada vaso recebeu $10 \mathrm{ml}$ de solução de Nitrato de Cálcio Pentahidratado (totalizando $87 \mathrm{mg}$ vaso $^{-1}$ de N e 125 mg vaso ${ }^{-1}$ de Ca). Aos 30, 40, 50 e 60 dias de cultivo da Brachiaria, também foram aplicados $10 \mathrm{ml} \mathrm{da}$ solução nutritiva de Hoagland e Arnon (1950).

Após três meses de cultivo armadilha, o solo dos vasos representantes de cada área em estudo foi homogeneizado e retirado $100 \mathrm{~cm}^{3}$ para a extração dos esporos por meio do método de peneiramento úmido (GERDEMANN; NICOLSON, 1963), e centrifugação em sacarose 40\% (JENKINS, 1964). Após a extração, foi realizada a contagem do número total de esporos em lupas binoculares com aumento de 40 vezes. Posteriormente, os esporos foram agrupados pelas características de tamanho, cor e forma, e colocados em lâminas com álcool polivinil em lactoglicerol (PVLG) sob uma lamínula. $\mathrm{Na}$ mesma lâmina um segundo grupo de esporos foi submetido a uma mistura de PVLG + Reagente de Melzer (1:1) e quebrados mecanicamente sob uma lamínula para exposição das paredes internas. A reação de cor ao reagente de Melzer foi utilizada para caracterizar as paredes dos esporos. A identificação dos gêneros e espécies de fungos micorrízicos arbusculares foi realizada pela análise das características morfológicas externas e internas dos esporos, conforme INVAM (2013). Após a identificação e contagem (abundância) dos táxons, as comunidades de FMAs foram caracterizadas pelos seguintes índices de diversidade: riqueza de Margalef, equitabilidade de Pielou, dominância de Simpson e diversidade de Shannon, conforme Odum (1986).

Uma amostra de solo das áreas analisadas foi utilizada para a determinação dos teores de Matéria Orgânica (M.O.), Fósforo (P) (Mehlich-1) e pH conforme Tedesco et al. (1995), para estudos de correlações.

Os dados foram submetidos à análise de variância (ANOVA) para detectar diferenças significativas entre os diferentes usos do solo. As médias foram comparadas pelo teste de ScottKnott a 5\% de significância, utilizando o programa estatístico SISVAR (FERREIRA, 2008). Análises de correlação linear de Pearson (r) foram executadas entre as os teores de M.O., P e pH com as espécies de FMAs encontradas nas diferentes áreas do presente trabalho. O estudo da relação entre a distribuição de esporos de FMAs e as áreas foi realizado por meio de uma ordenação gerada pela análise de correlação canônica (CCA), utilizando-se o software CANOCO Versão 4.0 (TER BRAAK; SMILAUER, 1998). 


\section{Resultados e Discussão}

Os resultados da extração de esporos de FMAs revelaram variação na ocorrência de Glomus mosseae, Glomus aggregatum, Glomus clarum, Acaulospora sp. e Scutellospora reticulata nas diferentes áreas avaliadas. O gênero Glomus foi o mais abundante, com maior número de esporos para a espécie $G$. mosseae, exceto no pousio no qual o G. clarum foi predominante (Tabela 1). O Glomus parece ser um dos gêneros com maior frequência de ocorrência no Brasil, sendo relatado em vários estudos sobre levantamento de FMAs (AZEVEDO et al., 2014; MIRANDA; SILVA; SAGIN JÚNIOR, 2010; MIRANDA et al., 2011; SILVA et al., 2012). A maior ocorrência neste levantamento do gênero Glomus pode estar relacionada ao caráter generalista quanto ao hospedeiro e por esse isolado ser capaz de colonizar maior número de espécies, facilitando seu estabelecimento na área (POUYU-ROJAS; SIQUEIRA; SANTOS, 2006). O predomínio do gênero Glomus também foi verificado por Leal, Stürmer e Siqueira (2009) em culturas armadilha de solos sob diferentes sistemas de uso na Amazônia.

Tabela 1. Número de esporos de fungos micorrízicos arbusculares (FMAs) e abundância de esporos em solo nas áreas estudadas, 2013.

\begin{tabular}{|c|c|c|c|c|c|c|}
\hline ÁREA & $\begin{array}{c}\text { Acaulospora } \\
\text { sp. }\end{array}$ & $\begin{array}{c}\text { Glomus } \\
\text { aggregatum }\end{array}$ & $\begin{array}{l}\text { Glomus } \\
\text { clarum }\end{array}$ & $\begin{array}{c}\text { Glomus } \\
\text { mosseae }\end{array}$ & $\begin{array}{l}\text { Scutellospora } \\
\text { reticulata }\end{array}$ & Abundância \\
\hline \multicolumn{7}{|c|}{ - - } \\
\hline $\mathrm{FN}^{* *}$ & $15 \mathrm{cD}^{*}$ & $94 \mathrm{dC}$ & $156 \mathrm{bB}$ & $248 \mathrm{dA}$ & $74 \mathrm{aC}$ & $587 \mathrm{~d}$ \\
\hline $\mathrm{P}$ & $43 \mathrm{bC}$ & $53 \mathrm{dC}$ & $270 \mathrm{aA}$ & $150 \mathrm{eB}$ & $0 \mathrm{dD}$ & $516 \mathrm{~d}$ \\
\hline PI & $2 \mathrm{dD}$ & $77 \mathrm{dC}$ & $162 \mathrm{bB}$ & $795 \mathrm{bA}$ & $6 \mathrm{cD}$ & $1042 \mathrm{~b}$ \\
\hline PE & $38 \mathrm{bD}$ & $420 \mathrm{bB}$ & $291 \mathrm{aC}$ & $555 \mathrm{cA}$ & $6 \mathrm{cE}$ & $1310 \mathrm{a}$ \\
\hline PD & $15 \mathrm{cD}$ & $255 \mathrm{cB}$ & $152 \mathrm{bC}$ & $990 \mathrm{aA}$ & $12 \mathrm{cD}$ & $1424 \mathrm{a}$ \\
\hline $\mathrm{PC}$ & $23 \mathrm{bD}$ & $244 \mathrm{cB}$ & $98 \mathrm{cC}$ & $692 \mathrm{cA}$ & $0 \mathrm{dE}$ & $1057 \mathrm{~b}$ \\
\hline PL & $8 \mathrm{cD}$ & $606 \mathrm{aA}$ & $85 \mathrm{cB}$ & $636 \mathrm{cA}$ & $30 \mathrm{bC}$ & $1365 \mathrm{a}$ \\
\hline $\mathrm{V}$ & $0 \mathrm{dE}$ & $113 \mathrm{~dB}$ & $49 \mathrm{dC}$ & $546 \mathrm{cA}$ & $8 \mathrm{cD}$ & $716 \mathrm{c}$ \\
\hline Ed & $66 \mathrm{aD}$ & $111 \mathrm{dC}$ & $152 \mathrm{bB}$ & $921 \mathrm{aA}$ & $0 \mathrm{dE}$ & $1250 \mathrm{a}$ \\
\hline
\end{tabular}

* Médias seguidas de mesma letra minúscula na coluna e maiúscula na linha não diferem entre si pelo teste de Scott-Knott a 5\% de probabilidade.

**Floresta nativa (FN); pousio (P); pastagem intensiva (PI); pastagem extensiva (PE); plantio direto (PD); plantio convencional (PC); pomar de laranja (PL); videira (V); Eucalyptus dunnii (Ed).

Fonte: Elaboração dos autores.

$\mathrm{Na}$ área com videira não foi identificado esporos de Acaulospora, enquanto que na área com Eucalyptus dunnii evidenciou-se maior abundância para este gênero de FMA, não diferindo estatisticamente da área em pousio (Tabela 1). Resultados similares foram obtidos por Mello et al. (2006) e Araújo (2008) no qual o gênero Acaulospora apresentou maior abundância de esporos na área com eucalipto, áreas com processo de recuperação e em campo nativo.
O número de esporos de G. aggregatum foi maior no pomar de laranja (Tabela 1). Esse resultado diverge de Souza et al. (2002) que embora tenham identificados cinco espécies do gênero Glomus, em viveiros e pomares de citrus no Rio Grande do Sul, não identificaram esporos do G. aggregatum. O número de esporos do G. clarum foi maior nas áreas em pousio e com a pastagem extensiva, e o de G. mosseae no sistema plantio direto e Eucalyptus dunnii. Resultados de pesquisa têm relatado que 
entre os FMAs, os pertencentes à família Glomaceae ocorrem com maior frequência em diferentes solos, sugerindo melhor adaptação ao ambiente (MELLO et al., 2012).

Não foram identificados esporos de $S$. reticulata nas áreas em pousio, plantio convencional e Eucalyptus dunnii, sendo o número de esporos significativamente maior na floresta nativa em relação às demais áreas (Tabela 1). De acordo com Zangaro, Bononi e Trufen (2000), quando uma floresta é perturbada, ocorre diminuição ou eliminação das espécies de FMAs mais adaptadas aos hospedeiros e ao ambiente natural, ocorrendo uma seleção das espécies que se adaptam ao novo ambiente, refletindo também a compatibilidade de hospedeiros.

A abundância de esporos foi significativamente maior no plantio direto, pomar de laranja, pastagem extensiva e Eucalyptus dunnii e menor nas áreas de pousio e floresta nativa (Tabela 1). Angelini et al. (2012) também verificaram efeito da cultura de cobertura sobre o número de esporos de FMAs. Cordeiro et al. (2005) encontraram maior número de esporos de FMA sob pastagem natural degradada e em processo de reabilitação quando comparado ao cerrado sem a interferência humana. Conforme Poggiani e Schumacher (2000), em ecossistemas naturais, a ciclagem de nutrientes pode ser mais intensa e, consequentemente, o estímulo para ocorrência das interações micorrízicas com seus hospedeiros pode ser reduzido.

A análise multivariada evidenciou variação quanto aos FMAs em relação às áreas estudadas (Figura 1). Ao longo da dimensão 1 (eixo principal), as áreas de pousio, Eucalyptus dunnii e pastagem extensiva encontram-se dispostas na porção à direita (com valores positivos), as áreas de floresta nativa e plantio convencional na porção mediana, enquanto pastagem intensiva, videira, pomar de laranja e plantio convencional na porção à esquerda (com valores negativos). Desse modo, observa-se maior associação de G. mosseae e G. aggregatum na área de plantio direto e, Acaulospora sp. nas áreas de Eucalyptus dunnii e pastagem extensiva (Figura 1). Contudo, a espécie G. clarum apresentou menor tendência de associação com as diferentes áreas estudadas, enquanto as áreas de pousio, floresta nativa, plantio convencional e pomar de laranja não apresentaram associação com os FMAs (Figura 1). Staddon et al. (2003) afirmam que a ocorrência e a densidade de FMAs dependem da planta hospedeira, do fungo e de fatores edafoclimáticos. O conhecimento sobre a ocorrência dos FMAs nas diferentes áreas avaliadas pode servir de subsídios para futuras opções de uso do solo, pois conforme Melloni et al. (2000), esses fungos podem ser utilizados como bioindicadores da qualidade em função do manejo aplicado sobre o solo.

As espécies de $G$. clarum e $S$. reticulata apresentaram correlação de Pearson positiva de 0,83 e 0,84 nos solos em pousio e pastagem intensiva com baixo teor de matéria orgânica, respectivamente 2,2 e 2,1\% (Tabela 2). Entretanto, na floresta nativa em solo com 4,2 \% de matéria orgânica e na pastagem extensiva com $3,1 \%$ de matéria orgânica as espécies de G. clarum e S. reticulata apresentaram correlação negativa de 0,97 e 0,85 , respectivamente. Benedetti et al. (2005) verificaram variação na abundância de esporos de espécies de FMAs e tendência de diminuição em solos com teores elevados de matéria orgânica. Os resultados obtidos neste trabalho concordam com os de Benedetti et al. (2005), pois também indicam correlação negativa de G. clarum e $S$. reticulata com maiores teores de matéria orgânica do solo.

Em geral, solos com teores de fósforo menores que $40 \mathrm{mg} \mathrm{dm}^{-3}$ favorecem a ocorrência de espécies de FMAs (COLLINS; FOSTER, 2009) e em solos com concentração de fósforo maior que $40 \mathrm{mg}$ $\mathrm{dm}^{-3}$ a esporulação e a diversidade de FMA são negativamente afetados (GAI et al., 2009). Apesar dos teores de fósforo nas áreas do presente estudo

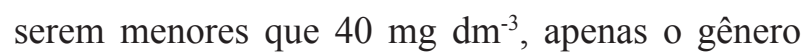
Glomus apresentou alta correlação com os teores de fósforo nas áreas sob pousio e plantio direto. 
Figura 1. Representação gráfica da análise de correlação canônica (CCA) entre os esporos de Glomus mosseae, Glomus aggregatum, Glomus clarum, Acaulospora sp. e Scutellospora reticulata em solos com floresta nativa (FN); pousio (P); pastagem intensiva (PI); pastagem extensiva (PE); plantio direto (PD); plantio convencional (PC); pomar de laranja (PL); videira (V); Eucalyptus dunnii (Ed). 2013.

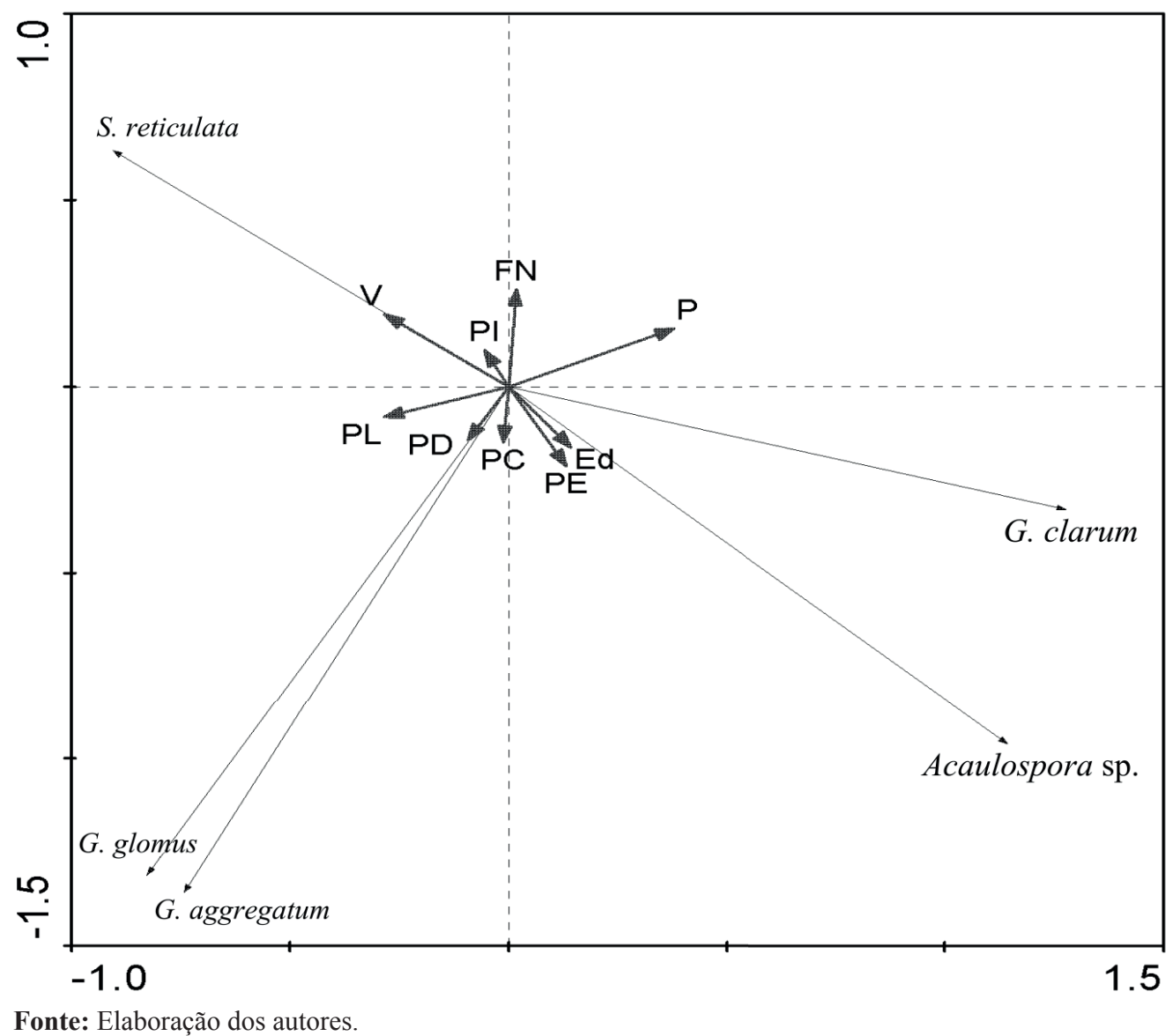

Todas as espécies de FMAs apresentaram correlação alta com o pH do solo em uma das áreas avaliadas. O gênero Acaulospora apresentou correlação positiva $(0,87)$ para o $\mathrm{pH} 5$ na área de floresta nativa e correlação negativa $(-0,99)$ para o pH 6,6 no pomar de laranja (Tabela 2). De acordo Stürmer e Bellei (1994), o gênero Acaulospora é frequentemente encontrado em solos com $\mathrm{pH}$ menor que 6,2. Os resultados do presente estudo, também evidenciam que o gênero Acaulospora é favorecido em ambientes com pH inferior a 6,2.

As espécies G. aggregatum e $G$. clarum apresentaram correlações negativas com $\mathrm{pH}$ ácido $(5,0$ e 5,5) em floresta nativa e área de pousio, respectivamente, positiva no pomar de laranja com
pH 6,6, enquanto a espécie $S$. reticulata apresentou alta correlação em área sob floresta nativa e pastagem extensiva com $\mathrm{pH}$ de 5,0 e 5,5 respectivamente (Tabela 2). Costa et al. (2013) encontraram maior produção de esporos de G. clarum em $\mathrm{pH} \mathrm{4,0} \mathrm{em}$ condições controladas de laboratório, em raízes de cenoura. Ferreira, Carneiro e Saggin Junior (2012) não evidenciaram correlação entre o $\mathrm{pH}$ e a densidade de esporos de FMAs em cinco áreas com diferentes uso do solo de um Latossolo Vermelho distroférrico. Entretanto, os resultados deste trabalho corroboram com os de Silveira (1998), os quais verificaram que valores de $\mathrm{pH}$ próximo da neutralidade favorece as espécies do gênero Glomus, enquanto que $\mathrm{pH}$ entre 4,0 e 6,0 as do gênero Scutellospora. 
Tabela 2. Correlação linear de Pearson (r) entre os Teores de Matéria Orgânica (M.O.: \%), Fósforo (P: mg dm-3), e pH do solo, com a ocorrência das espécies de FMAs (G. = Glomus; Sc. $=$ Scutellospora $) .2013$.

\begin{tabular}{|c|c|c|c|c|c|c|c|}
\hline Área & \multicolumn{2}{|c|}{ Teores } & $\begin{array}{l}\text { Acaulospora } \\
\text { sp. }\end{array}$ & $\begin{array}{c}G . \\
\text { aggregatum }\end{array}$ & $\begin{array}{c}\text { G. } \\
\text { clarum }\end{array}$ & $\begin{array}{c}G . \\
\text { mosseae }\end{array}$ & $\begin{array}{c}\text { Sc. } \\
\text { reticulata }\end{array}$ \\
\hline \multirow{3}{*}{$\mathrm{FN}^{* * *}$} & M.O. & 4,2 & $0,30 *$ & 0,00 & $-0,97$ & 0,11 & 0,48 \\
\hline & $\mathrm{P}$ & 2 & 0,00 & 0,27 & $-0,43$ & 0,00 & $-0,35$ \\
\hline & $\mathrm{pH}$ & 5 & 0,87 & $-0,96$ & $-0,21$ & $-0,76$ & 0,84 \\
\hline \multirow{3}{*}{$\mathrm{P}$} & M.O. & 2,2 & 0,48 & $-0,17$ & 0,83 & 0,70 & $* *$ \\
\hline & $\mathrm{P}$ & 14,4 & $-0,71$ & 0,95 & $-0,07$ & $-0,42$ & $* *$ \\
\hline & $\mathrm{pH}$ & 5,5 & $-0,48$ & 0,20 & $-0,84$ & $-0,70$ & $* *$ \\
\hline \multirow{3}{*}{ PI } & M.O. & 2,1 & 0,00 & 0,09 & $-0,55$ & $-0,56$ & 0,85 \\
\hline & $\mathrm{P}$ & 17 & 0,00 & $-0,10$ & 0,60 & 0,52 & $-0,76$ \\
\hline & $\mathrm{pH}$ & 5,7 & 0,00 & $-0,09$ & 0,58 & 0,60 & $-0,78$ \\
\hline \multirow{3}{*}{ PE } & M.O. & 3,1 & 0,70 & 0,10 & 0,42 & 0,36 & $-0,85$ \\
\hline & $\mathrm{P}$ & 6,5 & 0,69 & 0,66 & $-0,88$ & 0,08 & 0,45 \\
\hline & $\mathrm{pH}$ & 5,4 & $-0,77$ & $-0,10$ & $-0,46$ & $-0,36$ & 0,85 \\
\hline \multirow{3}{*}{ PD } & M.O. & 2 & 0,47 & 0,36 & $-0,13$ & $-0,40$ & 0,77 \\
\hline & $\mathrm{P}$ & 4 & 0,53 & $-0,54$ & 0,76 & 0,87 & $-0,71$ \\
\hline & $\mathrm{pH}$ & 6,2 & 0,00 & $-0,63$ & $-0,14$ & 0,43 & 0,00 \\
\hline \multirow{3}{*}{$\mathrm{PC}$} & M.O. & 1,9 & 0,00 & $-0,51$ & $-0,75$ & 0,08 & $* *$ \\
\hline & $\mathrm{P}$ & 6,3 & 0,00 & $-0,53$ & $-0,82$ & 0,05 & $* *$ \\
\hline & $\mathrm{pH}$ & 5,2 & 0,00 & 0,49 & 0,76 & $-0,06$ & $* *$ \\
\hline \multirow{3}{*}{$\mathrm{V}$} & M.O. & 2,9 & $* *$ & $-0,41$ & 0,34 & $-0,03$ & 0,00 \\
\hline & $\mathrm{P}$ & 9,7 & $* *$ & 0,15 & $-0,64$ & $-0,80$ & 0,53 \\
\hline & $\mathrm{pH}$ & 6,3 & $* *$ & $-0,27$ & $-0,30$ & $-0,84$ & 0,52 \\
\hline \multirow{3}{*}{ PL } & M.O. & 3 & 0,00 & 0,08 & 0,22 & 0,57 & 0,53 \\
\hline & $\mathrm{P}$ & 17,1 & $-0,53$ & 0,40 & 0,48 & $-0,28$ & $-0,47$ \\
\hline & $\mathrm{pH}$ & 6,6 & $-0,99$ & 0,98 & 0,96 & 0,04 & $-0,47$ \\
\hline \multirow{3}{*}{ ED } & M.O. & 2,7 & 0,26 & $-0,05$ & 0,51 & 0,68 & $* *$ \\
\hline & $\mathrm{P}$ & 2,7 & 0,52 & 0,69 & 0,10 & $-0,06$ & $* *$ \\
\hline & $\mathrm{pH}$ & 6,1 & $-0,26$ & 0,07 & $-0,53$ & $-0,69$ & $* *$ \\
\hline
\end{tabular}

*Coeficiente de correlação linear de Pearson (r);

** Não encontrado esporos.

***Floresta nativa (FN); pousio (P); pastagem intensiva (PI); pastagem extensiva (PE); plantio direto (PD); plantio convencional (PC); pomar de laranja (PL); videira (V); Eucalyptus dunnii (Ed).

Fonte: Elaboração dos autores.

O G. mossae apresentou correlação negativa para o $\mathrm{pH}$ 6,3 na área de videira (Tabela 2). Nogueirol et al. (2010) relataram que as videiras são submetidas à aplicações sucessivas de fungicidas cúpricos foliares que ao longo do tempo causam acúmulo de cobre em solos de vinhedos. Atlas e Bartha (1998) afirmam que plantas, fungos e bactérias, submetidas a concentrações elevadas de cobre, passam por uma pressão de seleção, mantendo somente os organismos resistentes no local contaminado. Embora a diversidade e o número de esporos de FMAs possam ser influenciados pelos efeitos da matéria orgânica, $\mathrm{pH}$ e $\mathrm{P}$ do solo, há dificuldade em estabelecer uma relação clara entre as variáveis edáficas e a ocorrência e diversidade de FMAs (STÜRMER; SIQUEIRA, 2008). É possível que o G. mosseae apresente menor tolerância a teores mais elevados de cobre no solo, no entanto, essa hipótese deverá ser comprovada em outros trabalhos. 
No que se refere à análise da diversidade de Santos, Scoriza e Ferreira (2013) também obtiveram FMAs, verificou-se maior valor de riqueza de maior riqueza de espécies na floresta nativa, Margalef na área de floresta nativa, não diferindo podendo ser consequência da sua maior estabilidade estatisticamente do pousio, pastagem extensiva, e da menor competição por nichos, possibilitando a plantio direto e área com pomar de laranja (Figura 2). sobrevivência das espécies com baixa esporulação.

Figura 2. Riqueza de Margalef, Equitabilidade de Pielou, Dominância de Simpson e Diversidade de Shannon das espécies de fungos micorrízicos arbusculares em áreas com diferentes usos do solo, 2013.
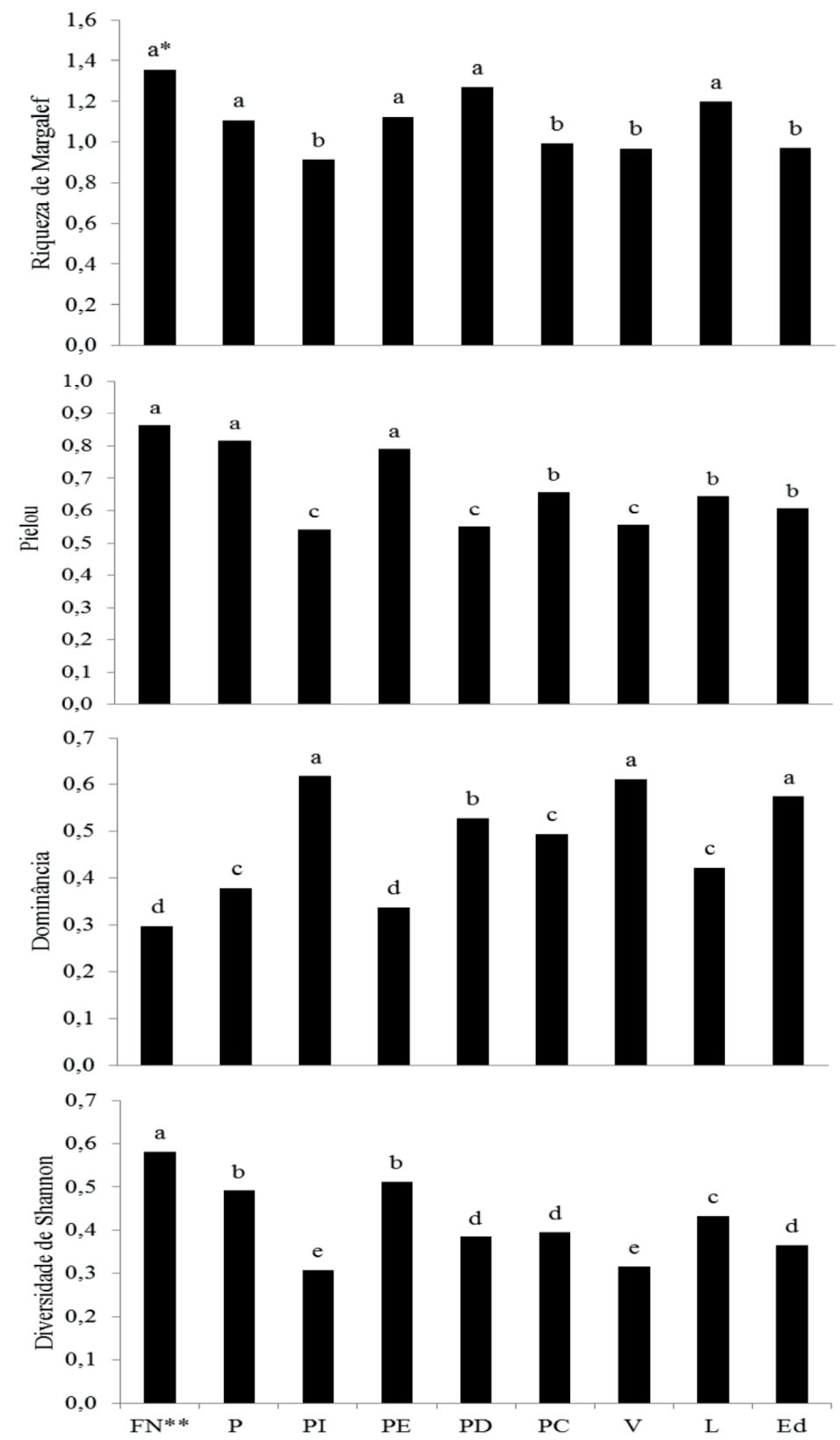

*Médias seguidas de mesma letra não diferem entre si pelo teste de Scott-Knott a 5\% de probabilidade. **Floresta nativa (FN); pousio (P); pastagem intensiva (PI); pastagem extensiva (PE); plantio direto (PD); plantio convencional (PC); videira (V); pomar de laranja (PL); Eucalyptus dunnii (Ed).

Fonte: Elaboração dos autores. 
$\mathrm{O}$ índice de uniformidade de Pielou foi maior nas áreas de floresta nativa, pousio e pastagem extensiva em comparação às demais áreas estudadas (Figura 2). Carneiro et al. (2012) obtiveram maior valor no índice de Pielou em área sob processo inicial de degradação, quando comparada a áreas degradadas em recuperação e floresta nativa. Desse modo, as possíveis alterações ocasionadas no solo pelos diferentes usos podem ter afetado a distribuição de espécies de FMAs influenciando neste índice de diversidade.

As áreas com pastagem intensiva, videira e Eucalyptus dunnii apresentaram maior valor para dominância de Simpson, enquanto a menor dominância de indivíduos ocorreu na floresta nativa e pastagem extensiva (Figura 2). Borba e Amorim (2007) evidenciaram maior dominância do gênero Glomus, sugerindo uma melhor adaptação ao $\mathrm{pH}$ do solo das áreas amostradas. Entretanto, houve baixa correlação entre o $\mathrm{pH}$ e o gênero Glomus nas áreas de pastagem intensiva, pomar de videira e Eucalyptus dunnii (Tabela 1), Isso pode ser um indício de favorecimento do uso dessas áreas para a ocorrência de determinada espécie de FMA, conforme observado para o Glomus mosseae (Tabela 1), resultando em maior dominância.

A diversidade de Shannon foi maior na floresta nativa e menor na pastagem intensiva e videira em comparação com as demais áreas (Figura 2). Conforme Ferreira, Carneiro e Saggin Junior (2012) a mudança no uso do solo promove alterações na comunidade de FMAs, podendo a densidade de esporos e diversidade dos fungos ser ampliadas, no caso da floresta nativa, ou reduzidas com monoculturas. É possível que sistemas não perturbados, por apresentarem diversidade na comunidade de plantas, possibilitem esporulação de diferentes espécies de FMAs.

\section{Conclusões}

O gênero Glomus é o mais abundante nas áreas de floresta nativa, pousio, pastagem intensiva, pastagem extensiva, plantio direto, plantio convencional, videira, pomar de laranja e Eucalyptus dunnii.

O plantio direto proporciona maior abundância de esporos de FMAs.

$\mathrm{O}$ pH do solo afeta a ocorrência de espécies de FMAs.

A floresta nativa apresentou menor dominância e maior diversidade de espécies de FMAs quando comparada às áreas com diferentes usos do solo.

\section{Agradecimentos}

ACAPES pela concessão das bolsas de Mestrado, a FAPERGS pelo auxilio financeiro e a UFSM pelos equipamentos e estrutura laboratorial.

\section{Referências}

ALVARES, C. A.; STAPE, J. L.; SENTELHAS, P. C.; GONÇALVES, J. L. M.; SPAROVEK, G. Köppen's climate classification map for Brazil. Meteorologische Zeitschrift, Stuttgart, v. 22, n. 6, p. 711-728, 2013.

ANGELINI, G. A. R.; LOSS, A.; PEREIRA, M. G.; TORRES, J. L. R.; SAGGIN JUNIOR, O. J. Colonização micorrízica, densidade de esporos e diversidade de fungos micorrízicos arbusculares em solo de Cerrado sob plantio direto e convencional. Semina: Ciências Agrárias, Londrina, v. 33, n. 1, p. 115-130, 2012.

ANTONIOLLI, Z. I. Arbuscular mycorrhizal community in a permanent pasture and development on the speciesspecific primers for detection and quantification of two AM fungi. 1999. Tese (Doutorado em Ciência do Solo) - Curso de Pós-Graduação em Ciência do Solo. The University of Adelaide, Adelaide.

ARAÚJO, F. S. Potencial de inoculo de fungos micorrízicos arbusculares em seis sistemas de uso do solo, na região nordeste do semiárido do Brasil, 2008. Dissertação (Mestrado em Zootecnia) - Universidade Federal de Campina Grande, Patos.

ATLAS, R. M.; BARTHA, R. Microbial ecology: fundamentals and applications. 4. ed. Menlo Park: Benjamin/Cummings Science Publishing, 1998. 694 p.

AZEVEDO, J. M. A. de; ASSIS, G. M. L. de; SAGGIN JUNIOR, O. J.; AZEVEDO, H. S. F. da S. Riqueza e frequência de espécies de fungos micorrízicos arbusculares em genótipos de amendoim forrageiro no 
Acre, Norte do Brasil. Acta Amazonica, Manaus, v. 44, n. 2, p. 157-168, 2014.

BALOTA, E. L.; MACHINESKI, O.; STENZ, N. Eficiência micorrízica em mudas de acerola sob diferentes níveis de fósforo. Synergismus Scyentifica, Pato Branco, v. 4, n. 1, p. 166-175, 2009.

BENEDETTI, T.; GIRACCA, E. M. N.; STEFFEN, R. B.; ANTONIOLLI, Z. I. Diversidade de fungos micorrízicos arbusculares na cultura do milho após o uso de espécies de plantas de cobertura. Revista de Ciências Agroveterinárias, Lages, v. 4, n. 1, p. 44-51, 2005.

BORBA, M. F.; AMORIM, S. M. C. de. Fungos micorrízicos arbusculares em sempre-vivas: subsídio para cultivo e replantio em áreas degradadas. Revista de Biologia e Ciências da Terra, Paraíba, v. 7, n. 2, p. 2027, 2007.

CARNEIRO, R. F. V.; CARDOZO JÚNIOR, F. M.; PEREIRA, L. F.; ARAÚJO, A. S. F.; SILVA, G. A. Fungos micorrízicos arbusculares como indicadores da recuperação de áreas degradadas no Nordeste do Brasil. Revista Ciência Agronômica, Fortaleza, v. 43, n. 4, p. 648-657, 2012.

COLLINS, C. D.; FOSTER, B. L. Community-level consequences of mycorrhizae depend on phosphorus availability. Ecology, Washington, v. 90, n. 9, p. 25672576, 2009.

CORDEIRO, M. A. S., CARNEIRO, M. A. C.; PAULINO, H. B.; SAGGIN JUNIOR, O. J. Colonização e densidade de esporos de fungos micorrízicos em dois solos do cerrado sob diferentes sistemas de manejo. Pesquisa Agropecuária Tropical, Goiânia, v. 35, n. 3, p. 147-153, 2005.

COSTA, F. A.; HADDAD, L. S. M.; KASUYA, M. C. M.; OTON, W. C.; COSTA, M. D.; BORGES, A. C. In vitro culture of Gigaspora decipiens and Glomus clarum in transformed roots of carrot: the influence of temperature and pH. Acta Scientiarum, Maringá, v. 35, n. 3, p. 315-323, 2013.

EMPRESA BRASILEIRA DE PESQUISA AGROPECUÁRIA - EMBRAPA. Centro Nacional de Pesquisa de Solos. Sistema brasileiro de classificação de solos. 2. ed. Rio de Janeiro: Embrapa solos, 2006. 306 p.

FERNANDES, R. A. Impacto de usos de um Latossolo Vermelho de cerrado sobre a diversidade de fungos micorrizicos arbusculares. 2009. Dissertação (Mestrado em Agronomia) - Universidade Federal de Goiás, Jataí.

FERREIRA, D. A.; CARNEIRO, M. A. C.; SAGGIN JUNIOR, O. J. Fungos micorrízicos arbusculares em Latossolo Vermelho sob manejo e usos no cerrado.
Revista Brasileira de Ciência do solo, Viçosa, MG, v. 36, n. 1, p. 51-61, 2012.

FERREIRA, D. F. SISVAR: um programa para análises e ensino de estatística. Revista Symposium, Lavras, v. 6, n. 2, p. 36-41, 2008.

GAI, J. P.; CHRISTIE, P.; CAI, X. B.; FAN, J. Q.; ZHANG, J. L.; FENG, G.; LI, X. L. Occurrence and distribution of arbuscular mycorrhizal fungal species in three types of grassland community of the Tibetan Plateau. Ecological Research, Beijing, v. 24, n. 6, p. 1345-1350, 2009.

GERDEMANN, J. W.; NICOLSON, T. H. Spores of mycorrhizal endogone species extracted from soil by wetsieving and decanting. Transactions of the British Mycological Society, Londres, v. 46, n. 2, p. 235-244, 1963.

GOMIDE, O. H. O. Diversidade de fungos micorrizicos arbusculares em diferentes fitofisionomias do Pantanal da Nhecolândia. 2013. Tese (Doutorado em Ciência do Solo) - Programa de Pós-Graduação em Ciência do Solo. Universidade Federal de Lavras, Lavras.

GUO, W.; ZHAOA, R.; ZHAOA, W.; FUA, R.; GUOA, J.; BIA, N.; ZHANGB, J. Effects of arbuscular mycorrhizal fungi on maize (Zea mays L.) and sorghum (Sorghum bicolor L. Moench) grown in rare earth elements of mine tailings. Applied Soil Ecology, Amsterdam, v. 72, n. 1, p. 85-92, 2013.

MELLO, A. H.; ANTONIOLLI, Z. I.; KAMINSKI, J.; SOUZA, E. L.; OLIVEIRA, V. L. Fungos arbusculares e ectomicorrízicos em áreas de Eucalipto e campo nativo em solo arenoso. Ciência Florestal, Santa Maria, v. 16, n. 3, p. 293-301, 2006.

HOAGLAND, D. R.; ARNON, D. I. The water culture method for growing plants without soils. Berkeley: California Agricultural Experimental Station, 1950. 347 p.

INTERNATIONAL CULTURE COLLECTION OF ARBUSCULAR AND VESICULAR MYCORRHIZAL FUNGI - INVAM. Classification of glomeromycota. West Virginia University: Morgantown, 2013. Available at: $\quad<$ http://invam.caf.wvu.edu/fungi/taxonomy/ classification.htm>. Accessed at: 24 maio 2013.

JENKINS, W. R. A rapid centrifugal-flotation technique for separating nematodes from soil. Plant Disease, St. Paul, v. 48, n. 9, p. 692, 1964.

LEAL, P. L.; STÜRMER, S. L.; SIQUEIRA, J. O. Occurrence and diversity of arbuscular mycorrhizal fungi in trap cultures from soils under different land use systems in the Amazon, Brazil. Brazilian Journal of Microbiology, São Paulo, v. 40, n. 1, p. 111-121, 2009. 
MELLO, C. M. A.; SILVA, I. R.; PONTES, J. S.; GOTO, B. T.; SILVA, G. A.; MAIA, L. C. Diversidade de fungos micorrízicos arbusculares em área de Caatinga, PE, Brasil. Acta Botanica Brasilica, São Paulo, v. 26, n. 4, p. 938-943, 2012.

MELLONI, R.; NOGUEIRA, M. A.; FREIRE, V. F.; CARDOSO, E. J. B. N. Fósforo adicionado e fungos micorrizicos arbusculares no crescimento e nutrição mineral de limoeiro-cravo (Citruslimonia (L) Osbeck). Revista Brasileira de Ciência do Solo, Viçosa, MG, v. 24, n. 4, p. 767-775, 2000.

MENDES, I. de C.; REIS JUNIOR, F. B. Microrganismos do solo a e sustentabilidade dos agroecossistemas. Planaltina: Embrapa Cerrados, 2010. Disponível em: $<$ http://www.cpac.embrapa.br/noticias/artigosmidia/ publicados/188/>. Acesso em: 22 maio 2013.

MIRANDA, E. M.; SILVA, E. M. R.; SAGIN JÚNIOR, O. J. Comunidades de fungos micorrízicos arbusculares associados ao amendoim forrageiro em pastagens consorciadas no Estado do Acre, Brasil. Acta Amazonica, Manaus, v. 30, n. 1, p. 13-22, 2010.

MIRANDA, P. B.; MELLO, A. H.; PEREIRA, F. D.; MANESCHY, R. Q. Distribuição de inóculo de fungos micorrízicos arbusculares para sistemas agroflorestais na agricultura familiar. Agroecossistemas, Marabá, v. 3, n. 1, p. 45-51, 2011.

NOGUEIROL, R. C.; ALLEONI, L. R. F.; NACHTIGALL, G. R.; MELO, G. W. Sequential extraction and availability of copper in $\mathrm{Cu}$ fungicideamended vineyard soils from Southern Brazil. Journal of Hazardous Materials, Amsterdam, v. 181, n. 1-3, p. 931-937, 2010.

ODUM, E. P. Ecologia. Rio de Janeiro: Ed. Guanabara, 1986. $434 \mathrm{p}$.

POGGIANI, F.; SCHUMACHER, M. V. Ciclagem de nutrientes em florestas nativas. In: GONÇALVES, J. L. M.; BENEDETTI, V. Nutrição e fertilização florestal. Piracicaba: IPEF, 2000. p. 287-308.

POUYU-ROJAS, E.; SIQUEIRA, J. O.; SANTOS, J. G. D. Compatibilidade simbiótica de fungos micorrízicos arbusculares com espécies arbóreas tropicais. Revista Brasileira de Ciência do Solo, Viçosa, MG, v. 30, n. 3, p. 413-424, 2006.

RAMOS, M. L. G.; KONRAD, M. L. F.; SILVA, D. E.; RIBEIRO JÚNIOR, W. Q.; BATISTA, L. M. T. Diversidade de fungos micorrízicos e colonização radicular, em forrageiras solteiras e em consórcio com milho. Bioscience Journal, Uberlândia, v. 28, n. 2, p. 235-244, 2012.
SANTOS, R. S.; SCORIZA, R. N.; FERREIRA, J. $\mathrm{S}$. Fungos micorrízicos arbusculares em diferentes coberturas florestais em Vitória da Conquista, Bahia. Floresta Ambiente, Seropédica, v. 20, n. 3, p. 344-350, 2013.

SCHIAVO, J. A.; MARTINS, M. A. Produção de mudas de goiabeira (Psidium guajava IL.), inoculadas com o fungo micorrízico arbuscular Glomus clarum, em substrato agroindustrial. Revista Brasileira de Fruticultura, Jaboticabal, v. 24, n. 2, p. 519-523, 2002.

SILVA, C. F.; SIMÕES-ARAÚJO, J. L.; SILVA, E. M. R.; PEREIRA, M. G.; FREITAS, M. S. M.; SAGGIN JÚNIOR, O. J.; MARTINS, M. A. fungos micorrízicos arbusculares e proteína do solo relacionada à glomalina em área degradada por extração de argila e revegetada com eucalipto e acácia. Ciência Florestal, Santa Maria, v. 22, n. 4, p. 749-761, 2012.

SILVEIRA, A. P. D. Ecologia de fungos micorrízicos arbusculares. In: MELO, J. L. (Ed.). Ecologia microbiana. Jaguariúna: Embrapa, 1998. p. 61-83.

SMITH, S. E.; READ, D. J. Mycorrhizal symbiosis. London: Academic Press, 2008. 800 p.

SOUZA, P. V. D.; SCHMITZ, J. A. K.; FREITAS, R. S.; CARNIEL, E.; CARRENHO, R. Identificação e quantificação de fungos micorrízicos arbusculares autóctones em municípios produtores de citros no Rio Grande do Sul. Pesquisa Agropecuária Brasileira, Brasília, v. 37, n. 4, p. 553-558, 2002.

STADDON, P. L.; THOMPSON, K.; JAKOBSEN, I.; GRIME, J. P.; ASKEW, A. P.; FITTER, A. H. Mycorrhizal fungi abundance as affected by long-term climatic manipulations in the field. Global Change Biology, Urbana, v. 9, n. 2, p. 186-194, 2003.

STÜRMER, S. L.; BELLEI, M. M. Composition and seasonal variation of spore populations of arbuscular mycorrhizal fungi in dune soils on the Island of Santa Catarina, Brazil. Canadian Journal of Botany, Ottawa, v. 72, n. 3, p.359-363, 1994.

STÜRMER, S. L.; SIQUEIRA, J. O. Diversidade de fungos micorrízicos arbusculares em ecossistemas brasileiro. In: MOREIRA, F. M. S.; SIQUEIRA, J. O.; BRUSSARD, L. (Ed.). Biodiversidade do solo em ecossistemas brasileiros. Lavras: Universidade Federal de Lavras, 2008. p. 537-584.

TEDESCO, M. J.; GIANELlO, C.; BISSANI, C. A.; BOHNEN, H.; VOLKWEISS, S. J. Análise de solos, plantas e outros materiais. 2. ed. Porto Alegre: Universidade Federal do Rio Grande do Sul, 1995. 174 p. 
TER BRAAK, C. J. F.; SMILAUER, P. CANOCO reference manual and user's guide to canoco for windows: software for canonical community ordination (version 4). New York: Microcomputer Power, 1998. 351 p.
ZANGARO, W.; BONONI, V. L. R.; TRUFEN, S. B. Mycorrhizal dependency, inoculum potential and habitat preference of native woody species in South Brazil. Journal of Tropical Ecology, Winchelsea, v. 16, n. 4, p. 603-622, 2000. 